$16^{\text {th }}$ International Congress of Metrology, 05001 (2013)

DOI: $10.1051 /$ metrology/201305001

(C) Owned by the authors, published by EDP Sciences, 2013

\title{
Calibration of dosimeters following standard NF EN ISO 8041
}

\author{
Claire Bartoli ${ }^{1}$, Philippe Averlant2, Patrick Cellard $1^{1}$ \\ ${ }^{1,3}$ LNE, 1 rue Gaston Boissier, 75015 Paris, France ; ${ }^{2} 29$ rue Roger Hennequin 78197 Trappes, France
}

\begin{abstract}
In order to protect from occupational disease related to vibrations, the European Directive2002/44/CE defines alert and action limits which trigger an action on the vibrations received by the workers. Since the 6 th July 2010 , companies have been compelled to assess vibration doses thanks to a vibration dosimeter.LNE has been willing to develop its activities in accelerometry, LNE developed the calibration of vibration dosimeter service following standard ISO 8041 "Human Response to vibration / measuring instrumentation". This article describes the requirements of the European Directive 2002/44/CE, the standard ISO 8041 and the calibration bench developed and accredited by COFRAC.
\end{abstract}

\section{Introduction}

Environ un quart des salariés européen est exposé au moins $25 \%$ de son temps à des vibrations. Environ 200 cas de maladie professionnelle liés aux vibrations du système main bras ont été déclarées depuis les années 70 pour un coût moyen de 8,5 M€. De la même manière, 400 déclarations pour le système corps entier ont été faites avec des coûts d'indemnisation d'environ $12 \mathrm{M} €$.

$\mathrm{Ce}$ chiffre sous-estime sans doute très largement la réalité. En effet, les vibrations reçues par les travailleurs peuvent être à l' origine de troubles vertébraux (lombalgies, hernies discales), vasculaires (maladie des « doigts blancs »), neuropathiques (syndrome du canal carpien) ou ostéo-articulaire (tendinite).

Pour prévenir ces risques, la directive européenne 2002/44/CE, transposée en droit national en 2005, définit les valeurs limites d'exposition journalière (VLE) ainsi que les actions préventives à entreprendre à partir d'un certain niveau de vibration. Elle distingue deux types de vibrations :

- Les vibrations liées à la partie main-bras (MB ou HA en anglais) qui correspondent notamment à l'utilisation de marteaux piqueurs, clé à choc, ponceuses

Les vibrations liées à la partie corps entier (CE ou WB en anglais) qui correspondent par exemple à la conduite de chariots élévateurs, engins de BTP mais aussi matériel de jardinage.

\footnotetext{
a Corresponding author: author@email.org
}
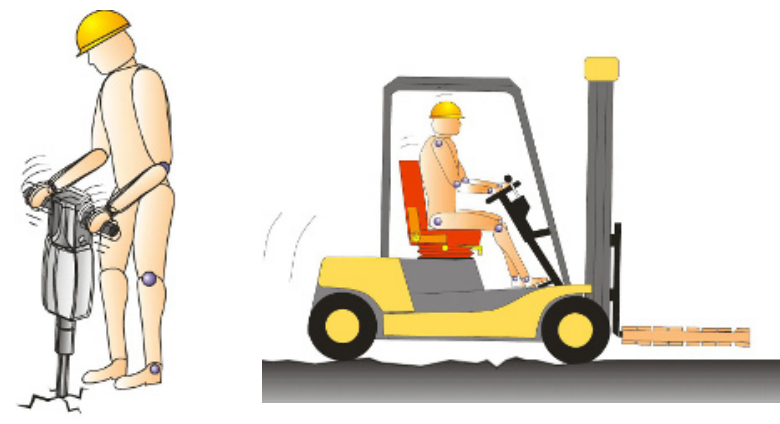

Figure 1. Illustration de cas de vibration corps entier et main bras

Il est donc nécessaire de pouvoir étalonner les matériels utilisés pour réaliser les mesures afin d'avoir confiance dans les valeurs fournies et de prendre les bonnes décisions. Jusqu'à présent, les étalonnages proposés en France ne couvraient pas les basses fréquences par manque de matériel adapté. Le LNE ayant étendu en 2010 le banc d'étalonnage des accéléromètres aux basses fréquences jusqu'à $0,5 \mathrm{~Hz}$, il est maintenant à même de répondre au besoin d'étalonnage des dosimètres vibratoires sur l'intégralité du domaine fréquentiel.

\section{Directive européenne 2002/44/CE}

Dans ce domaine de la santé au travail, il existe deux directives complémentaires qui imposent de mesurer les vibrations, la directive 2002/44/CE se place d'un point de vue de la dose reçue par le travailleur tandis que la 
directive 2006/42/Ce se place du coté de la dose émise par la machine.

1/ la directive européenne 2006/42/CE, liée à la sécurité des machines impose, aux fabricants de déclarer le niveau émis par une machine donnée. Ces mesures demandées par le fabricant sont réalisées en laboratoire extérieur ou en interne et peuvent s'éloigner des conditions réelles d'utilisation pour obtenir une meilleure définition et répétabilité de l'essai. Le mesurage et la déclaration des niveaux vibratoires n'est qu'une des nombreuses "exigences essentielles de sécurité et de santé". Ces mesures sont faite une fois au moment de la déclaration de conformité de la machine.

2/ La directive européenne 2002/44/CE concerne l'exposition réelle des travailleurs. Elle fixe des prescriptions minimales de sécurité et de santé relatives à l'exposition des travailleurs aux risques de vibration. On doit mesurer les vibrations au plus proche des conditions réelles d'utilisation et aux endroits où l'utilisateur est en contact avec la ou les machines.

La méthodologie et les moyens de mesure sont similaires dans les deux cas. La problématique métrologique et le besoin de raccordement sont donc les mêmes. Cette approche reproduit celle qui a été appliquée dans le cadre de la prévention des risques liés au bruit 2003/10/CE.

Ces directives s'appuient un contexte normatif complet afin de réaliser les mesures demandées. Les parties relatives aux méthodologies et aux matériels de mesure sont communes aux deux directives.

\section{Appareils de mesure}

Ces appareils de mesure sont prévus pour être utilisés par des non-spécialistes contrairement aux systèmes de mesure de vibrations habituels. Ils sont donc simples à utiliser et ergonomiques. Quelque soit le fabricant, l'appareil est composé d'un certain nombre de composants :

- Le boitier d'acquisition qui réalise le conditionnement des signaux, l'acquisition et les traitement à réaliser sur les signaux. Il peut se présenter sous différentes formes : avec ou sans affichage direct, avec une lecture indirecte sur un pocket pc, un ordinateur,...

\section{- Un accéléromètre} tri-axe dédié aux mesures pour le système main bras. Il est donc léger, peu encombrant et est fourni avec un certain nombre d'accessoires définis dans la norme ISO 5349-2.

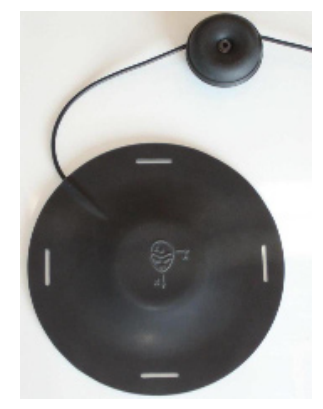

- Une assiette dans laquelle est intégré un capteur tri axial utilisé dans le cas des mesures corps entier. Elle peut être utilisée pour réaliser des mesures en position assise ou debout.

- Un accéléromètre mono axe réalisant la mesure du SEAT (Seat Effective Amplitude Transmissibility) peut également être utilisé. Il permet, en complément de l'assiette posée sur le siège, de calculer le coefficient de transmissibilité du siège en mesurant le niveau de vibration sur le plancher du véhicule.

L'utilisateur doit également avoir en sa possession un calibreur de vibration afin de vérifier le bon fonctionnement de sa chaine de mesure et de déterminer la sensibilité avant de réaliser les mesures (contrôle in situ). $\mathrm{Ce}$ calibreur doit bien entendu être étalonné régulièrement.

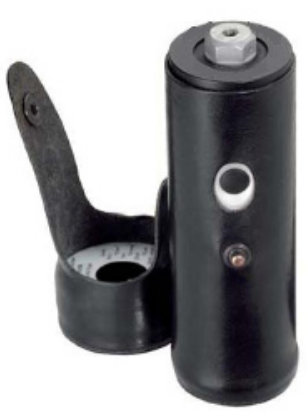

\section{Contexte normatif}

Afin de réaliser des mesures représentatives de la réalité et reproductibles, un certain nombre de normes apportent des méthodologies ainsi que des guides pratiques et techniques.

Les normes ISO 5349 et ISO 2631 décrivent les méthodes de mesurage des vibrations et d'évaluation.

La norme NF EN ISO 5349 : « mesurage et évaluation de l'exposition des individus aux vibrations transmises par la main » définit les exigences générales pour la mesure des vibrations ainsi que les aspects pratiques et les précautions à prendre pour réaliser ces mesures.

La norme NF EN ISO 2631 « évaluation de l'exposition des individus à des vibrations globales du corps » définit également, mais pour les mesures corps entier les exigences générales, les aspects pratiques et les précautions à prendre pour réaliser ces mesures pour le domaine corps entier.

La norme NF EN 30326-1 (ou ISO 10326-1) décrit plus spécifiquement la méthodologie de mesures des vibrations transmises par un siège et définit notamment le disque interface appelé communément galette dans lequel est intégré l'accéléromètre tri axe. Lors des essais réels, la personne "'objet de mesurage"' s'assoit sur cette galette pendant la conduite du tracteur ou tout autre engin afin de mesurer le niveau de vibration reçu par le conducteur. 


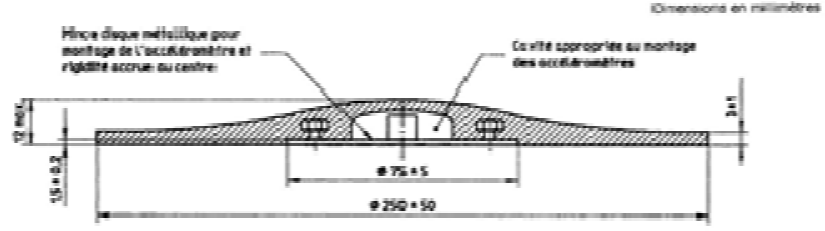

Figure 2. Schéma issu de la norme NF EN 30326

Une norme est par contre commune aux deux directives et aux deux domaines de mesure ainsi qu'à l'ensemble des normes du domaine, c'est la norme NF EN ISO 8041 " réponse des individus aux vibrations / appareillage de mesure ». Ces matériels peuvent avoir plusieurs dénominations : dosimètres vibratoires, exposimètre vibratoire, vibromètre, et en anglais human vibration analyser, vibration meter, HVM (human vibration meter ou monitor), ...

La norme définit les caractéristiques générales du produits (documentation, marquage de l'instrument, critères d'environnement). Elle décrit également les spécifications et les essais de performance auxquels doit répondre l'appareil.

La norme décrit trois niveaux d'essais :

- les essais de conformité destinés aux fabricants.

- les essais requis pour les vérifications périodiques destinés aux fabricants et aux utilisateurs.

- les essais a réaliser in situ destinés aux utilisateurs.

Le tableau suivant issu de la norme ISO 8041 récapitule l'ensemble des essais à réaliser dans chacun des cas.

Les essais de conformité ont pour objet de démontrer qu'un type d'instrument peut satisfaire aux spécifications définies dans la norme. Ils peuvent être utilisés pour les essais de type ou pour l'homologation des instruments. Ils ne sont, de fait, à réaliser qu'une fois.

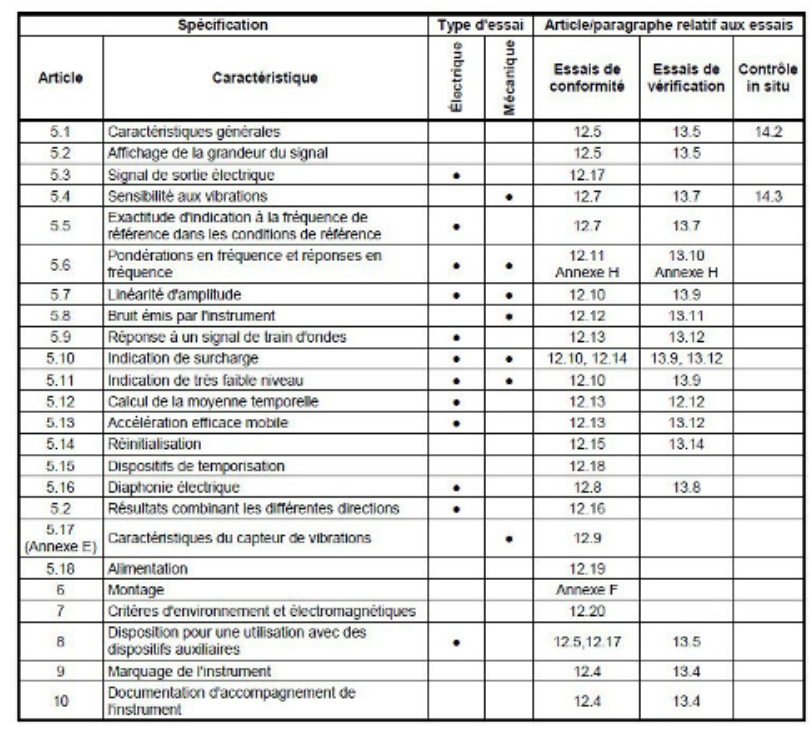

Figure 3. Tableau 13 de la norme ISO 8041
Les contrôles in situ se limitent à la vérification du bon fonctionnement de l'instrument avant utilisation et surtout de vérifier la sensibilité de l'instrument de mesure en utilisant un calibreur (ou calibrateur) de vibration.

Les essais de vérification correspondent aux essais à réaliser lors de l'étalonnage périodique de l'appareil afin d'en vérifier la conformité.

\section{Prestation d'étalonnage des dosimètres vibratoires développée par le LNE}

Dans le cadre du développement de ses activités et dans son souci de répondre aux besoins des industriels français, le LNE a décidé de proposer la prestation d'étalonnage des dosimètres vibratoires sous accréditation COFRAC. Le banc d'étalonnage des calibreurs existe déjà et est sous accréditation COFRAC.

Cette prestation suit donc les exigences du paragraphe 13 « essais de vérification » de la norme ISO 8041. Ce paragraphe liste un grand nombre d'essais qu'il est difficile et compliqué à réaliser lors d'un étalonnage annuel. Le LNE a donc fait le choix de ne réaliser qu'une partie des essais exigés par la norme et donc de ne pas déclarer la conformité des instruments à la norme dans les certificats d'étalonnage qu'il délivre.

Le document LAB REF 24 « Exigences spécifiques pour le mesurage de l'exposition aux vibrations en milieu de travail » révision 00 de Juillet 2009 disponible sur le site internet du COFRAC définit « les exigences à satisfaire par les organismes pour obtenir et maintenir l'accréditation pour les activités de mesurage de l'exposition aux vibrations en milieu de travail ».

Il exige au paragraphe 7.3.3 Traçabilité du mesurage que

- Pour les capteurs d'accélération, la traçabilité du mesurage doit être assurée sur l'ensemble du domaine fréquentiel d'utilisation conformément aux dispositions définies dans le LAB REF 02 (chapitre 9.3).

- Pour le système d'enregistrement et de traitement du signal, il convient a minima de s'assurer que les exigences de la norme NF EN ISO 8041 sont respectées en ce qui concerne :

- $\quad$ La linéarité d'amplitude ( $§ 13.9$ )

- La pondération en fréquences ( $\$ 13.10)$ pour les courbes utilisées dans le cadre des mesurages des vibrations

- $\quad$ L'indication de surcharge ( $§ 13.13)$

Conformément au LAB REF 24, le LNE a mis en place les essais électriques suivants :

- $\quad$ la détection de saturation (paragraphe 13.13)

- $\quad$ la linéarité d'amplitude (paragraphe 13.9)

- la réponse à un signal de train d'onde (paragraphe 13.12) 
Les essais électriques sont réalisés à la fréquence de référence soit $79,58 \mathrm{~Hz}$ pour le domaine main bras et $15.915 \mathrm{~Hz}$ pour le domaine corps entier.

En complément le LNE réalise l'essai électrique décrit au paragraphe 13.10.2 'essai mécanique de la réponse en fréquence » avec les pondérations dédiées (Wh pour le domaine main bras, $\mathrm{Wd}$ et $\mathrm{Wk}$ pour le domaine corps entier).

Les paragraphes suivants décrivent plus en détail le fonctionnement de chacun des essais métrologiques suivants.

Pour les essais électriques, un signal électrique est injecté en entrée du dosimètre. Un capteur virtuel est donc créé dans la configuration du dosimètre pour permettre de réaliser des mesures.

\section{Incertitudes d'étalonnage}

Les incertitudes d'étalonnage sur les caractéristiques métrologiques du dosimètre ne correspondent pas à celles données dans notre portée d'accréditation disponible sur le site du COFRAC.

Les incertitudes définies dans notre portée d'accréditation portent sur les signaux appliquées au dosimètres et non sur les valeurs indiquées par le dosimètre. En effet, certaines caractéristiques intrinsèques au dosimètre (résolution, dispersion...) ne permettent pas de prévoir les incertitudes sur les mesures lues sur le dosimètre.

Les deux informations vous seront données dans les paragraphes suivants.

\section{Linéarité d'amplitude}

Il s'agit de vérifier que le boitier donne une réponse proportionnelle au signal d'entrée quel que soit le niveau de celui ci. La sensibilité du boitier est déterminée à la fréquence de référence et au niveau de référence. Ensuite, on évalue l'écart à cette sensibilité de référence pour un signal d'entrée couvrant la gamme de fonctionnement de l'appareil. La norme ISO 8041 exige que le système présente une erreur de linéarité inférieure à $6 \%$ sur une gamme d'au moins $60 \mathrm{~dB}$ avec une incertitude maximale de $2 \%$.

Les incertitudes d'étalonnage estimées pour cet essai répond à ce critère excepté pour des niveaux de tension appliqués en entrée inférieur à $10 \mathrm{mV}$ rms.

L'incertitude élargie sur la tension appliquée est de 1,0\% pour une tension supérieure à $10 \mathrm{mVrms}$, et $2,5 \%$ pour une tension comprise entre 0,1 et $10 \mathrm{mVrms}$.

L'incertitude sur la linéarité d'amplitude du dosimètre prend également en compte les caractéristiques du dosimètre comme sa résolution, dispersion des valeurs affichées.

En prenant par exemple une résolution de 2 chiffres après la virgule, l'incertitude relative élargie $(\mathrm{k}=2)$ retenue sur la détermination des écarts de linéarité d'amplitude du dosimètre est prise égale à :

$2,0 \%$ avec $\mathrm{A} \geq 1 \mathrm{~m} / \mathrm{s}^{2}$

$4,0 \%$ avec $0,5 \leq \mathrm{A}<1 \mathrm{~m} / \mathrm{s}^{2}$

$9,0 \%$ avec $0,1 \leq \mathrm{A}<0,5 \mathrm{~m} / \mathrm{s}^{2}$

$20 \%$ avec $0,04 \leq \mathrm{A}<0,1 \mathrm{~m} / \mathrm{s}^{2}$

Des sensibilités théoriques de $10 \mathrm{mV} /\left(\mathrm{m} / \mathrm{s}^{2}\right)$ et 1 $\mathrm{mV} /\left(\mathrm{m} / \mathrm{s}^{2}\right)$ sont définies respectivement pour les domaines corps entier et main bras.

Si le comportement du dosimètre ne permet de répondre aux critères fixés dans le calcul d'incertitude en terme de dispersion, résolution ou autre, ces incertitudes peuvent être dégradées.

Les écarts de linéarité peuvent être supérieurs aux incertitudes à très bas niveau et donc être très supérieur à la tolérance. Cela ne porte pas à conséquence car, d'une part, les mesures se font à des niveaux qui correspondent au bruit de bond lorsque ce sont des mesures utilisant un accéléromètre et, d'autre part, car la norme exige une gamme de linéarité d'au moins $60 \mathrm{~dB}$ ce qui est le cas sans prendre en compte ces points à très bas niveau.

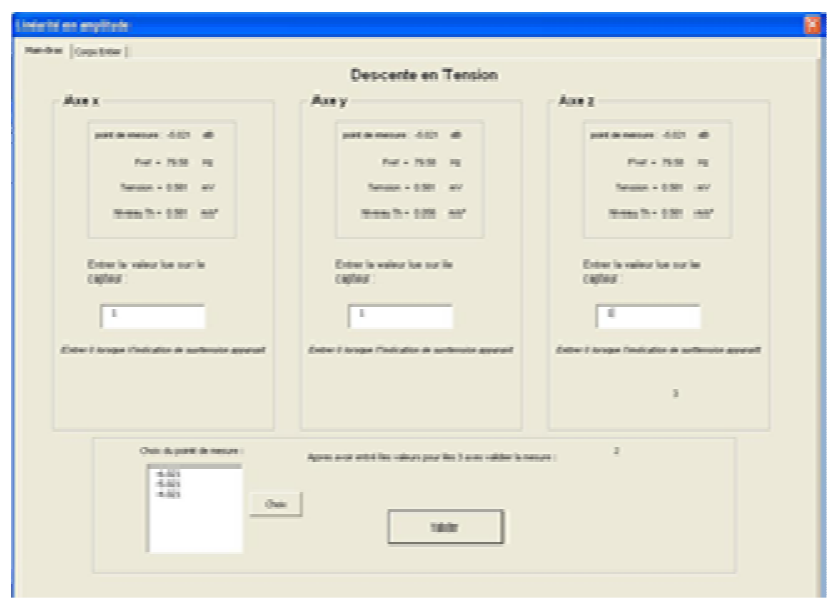

Figure 4. Copie d'écran du logiciel linéarité d'amplitude

\section{Détection de saturation}

Il s'agit de vérifier que le dosimètre alerte bien l'utilisateur lorsqu'une surcharge est appliquée à l'entrée $\mathrm{du}$ boitier. Cet essai se fait par application de signaux demi sinus positifs puis négatifs en augmentant le niveau jusqu'à indication de surcharge. L'écart entre les deux valeurs de signaux déclenchant l'alarme doit être inférieur à $15 \%$ avec une incertitude majorée maximale de $2 \%$. Cette incertitude élargie a été estimée à $1,5 \%$ dans le cadre de notre banc. La détection se faisant par diode luminescente, l'incertitude sur la tension appliquée est la même que celle sur la détection de surcharge par le dosimètre.

Le niveau déclenchant la surcharge peut varier d'un appareil ou d'une fois sur l'autre car il est fonction de la sensibilité théorique affectée au capteur virtuel. 


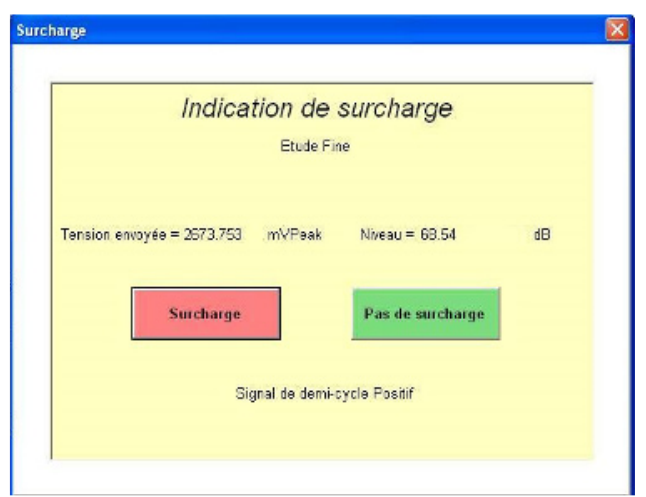

Figure 5. Copie d'écran du logiciel saturation

Réponse à un train d'onde

Un signal comprenant des trains de dents de scie est envoyé à un niveau connu en entrée et la réponse du dosimètre est comparée au résultat attendu.

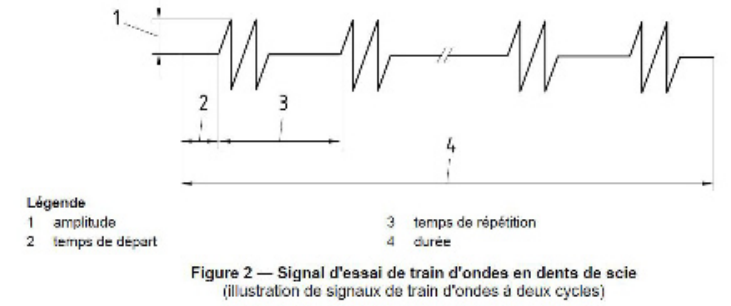

Figure 6. Signal issu de la norme ISO 8041

Ce signal contient des combinaisons de fréquences avec des relations de phases connues. L'essai du signal de train d'ondes en dents de scie assure par conséquent que le dosimètre traite correctement les signaux multi fréquence.

La tolérance sur l'écart entre la valeur lue et la valeur attendue est de $10 \%$ en intégrant une incertitude de mesure majorée maximale de $3 \%$.

Pour le LNE, l'incertitude élargie sur les signaux appliqués au dosimètre a été estimée à $2,5 \%$.

L'incertitude relative élargie $(\mathrm{k}=2)$ retenue pour la détermination de la réponse à un train d'onde du dosimètre est prise égale à $3,0 \%$.

\section{Essai mécanique de réponse en fréquence}

Cet essai mécanique, décrit dans le chapitre 13.10.2, permet de déterminer la réponse en fréquence de l'ensemble boîtier et accéléromètre par comparaison à une accélération de référence non pondérée. Celle-ci est déterminée à l'aide d'un accéléromètre étalon et d'un système d'acquisition.

L'erreur de réponse en fréquence donnée dans le certificat correspond à l'écart entre la valeur pondérée indiquée par le dosimètre et la valeur de vibration de référence multipliée par le facteur de pondération adéquat.

Avant de réaliser l'étalonnage mécanique du dosimètre, la valeur de la sensibilité de chacune des voies des capteurs est déterminée. Comme le requiert la norme lors d'une utilisation classique, la détermination est réalisée soit à l'aide d'un calibreur accélérométrique (typiquement à $159 \mathrm{~Hz}$ ) ou via la gravité pour les capteurs passant le continu.

Ces sensibilités sont ensuite entrées dans le dosimètre avant la détermination de la linéarité fréquentielle de celui-ci.

Le programme amplitude / fréquence est défini par la norme, Les fréquences nominales d'étalonnage sont les tiers d'octave de 0,5 à $160 \mathrm{~Hz}$ pour le domaine corps entier et de 8 à $2000 \mathrm{~Hz}$ pour le domaine main bras.

Le niveau d'étalonnage doit théoriquement être réglé 20 $\mathrm{dB}$ au-dessus de la limité inférieure de la plage de linéarité spécifiée. Ce n'est toutefois pas toujours le cas soit pour des raisons mécaniques (limites des excitateurs) soit parce que le signal lu sur le dosimètre se situe dans le bruit de fond (lié à une forte atténuation due à la pondération). L'essai métrologique est réalisé pour chacun des 3 axes de chaque capteur ainsi que pour le capteur mono axe SEAT.

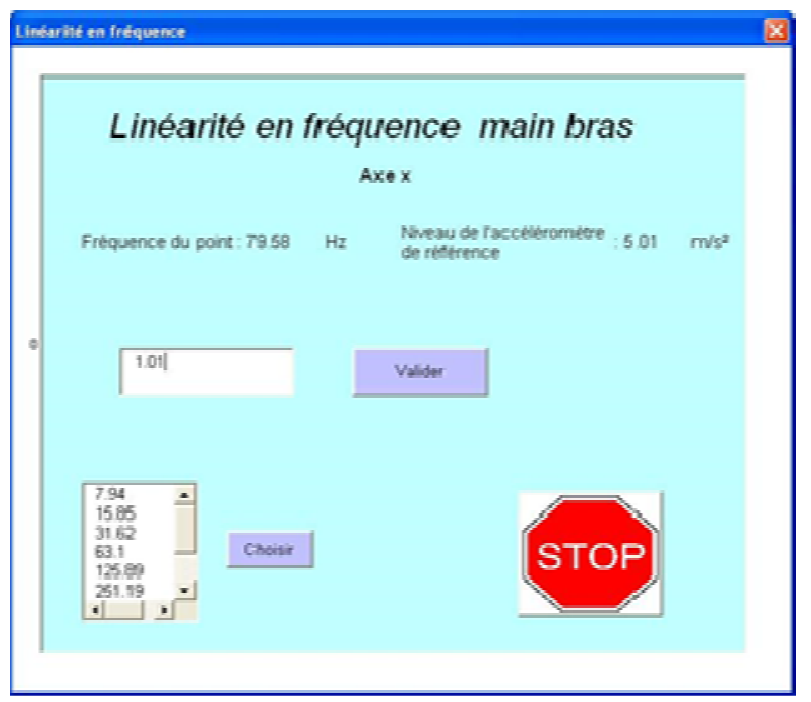

Figure 7. Copie d'écran du logiciel linéarité en fréquence

Les incertitudes de mesure majorées maximales requises par la norme sont de $5 \%$.

L'incertitude relative élargie $(\mathrm{k}=2)$ sur l'accélération appliquée est prise égale à :

$1,4 \%$ avec $\mathrm{A} \geq 1 \mathrm{~m} / \mathrm{s}^{2}$

$2,5 \%$ avec $0,2 \leq \mathrm{A}<1 \mathrm{~m} / \mathrm{s}^{2}$

Pour déterminer l'incertitude sur la linéarité fréquentielle $\mathrm{du}$ dosimètre, il faut intégrer, en complément de l'incertitude sur le niveau appliqué, les composantes liées à la mesure sur le dosimètre. Il s'agit notamment de la résolution de l'afficheur, de la dispersion des valeurs affichées et des caractéristiques des accéléromètres.

L'incertitude relative élargie $(\mathrm{k}=2)$ retenue sur la détermination de la linéarité fréquentielle du dosimètre en configuration main bras est prise égale à :

$2,0 \% \mathrm{Al}$ avec $\mathrm{Al} \leq 1 \mathrm{~m} / \mathrm{s}^{2}$

$5,0 \%$. Al avec $0,3 \leq \mathrm{Al}<1 \mathrm{~m} / \mathrm{s}^{2}$

$9,0 \% \mathrm{Al}$ avec $0,15 \leq \mathrm{Al}<0,3 \mathrm{~m} / \mathrm{s}^{2}$

$12,0 \% \mathrm{Al}$ avec $0,07 \leq \mathrm{Al}<0,15 \mathrm{~m} / \mathrm{s}^{2}$ 
$\mathrm{Al}$ : accélération lue sur le dosimètre

Des écarts de réponse en fréquence importants peuvent être constatés entre la réponse $\mathrm{du}$ dosimètre et l'accélération appliquée pondérée. Cela est dû la pondération qui peut atténuer le signal de 99,71\%.

Pour le domaine main bras, les dosimètres sont étalonnés avec les accéléromètres seuls c'est à dire sans aucun système de montage. Il est soit vissé sur l'accéléromètre de référence soit collé à la colle cyanolithe. Les systèmes de fixation ne sont utilisés lors de l'étalonnage.

Dans le capteur des accéléromètres pour le domaine corps entier, le capteur est démonté de la galette afin d'être étalonné dans les 3 axes.

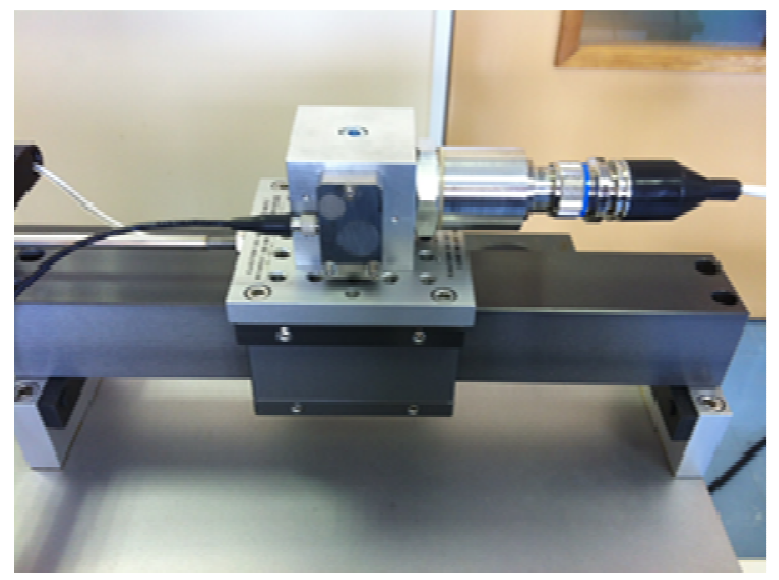

Figure 8. Montage d'un accéléromètre tri axe pour étalonnage dans l'axe $\mathrm{x}$ en mode corps entier

\section{Documents émis à l'issue de la prestation}

Le document émis à l'issue de cette prestation est un certificat d'étalonnage. Le LNE ne fournit pas de constat de vérification car tous les essais décris dans la norme ISO 8041 ne sont réalisés.

Les tolérances spécifiées dans l'annexe B de la norme sont indiquées pour chaque essais réalisé. Elles sont à mettre en rapport avec les incertitudes déclarées. Dans certains cas, les incertitudes de mesure sont supérieures aux tolérances définies par la norme, celles-ci ne prenant pas en compte les effets liés aux niveaux très faibles.

Par ailleurs, les essais concernant le domaine main bras et le domaine corps entier étant totalement découplés, l'utilisateur peut faire étalonner tant via les méthode mécanique qu'électrique, son dosimètre en mode corps entier ou en mode main bras.

\section{Conclusion}

Cette problématique de santé publique est maintenant bien prise en compte au sein des entreprises françaises. Le nouveau banc d'étalonnage des dosimètres du LNE va permettre de répondre à la demande d'étalonnage sous accréditation COFRAC des entreprises et des différents organismes à même de réaliser ces estimations des doses de vibrations reçues afin de mettre en place les actions préventives et correctives nécessaires pour protéger la santé des personnes.

\section{References}

Norme NF EN ISO 8041 : 2005 «Réponse des individus aux vibrations ; Appareillage de mesure »

Norme NF EN ISO 5349 -1 \& -2 « mesurage et évaluation de l'exposition des individus aux vibrations transmises par la main »; partie 1 exigences générales ; partie 2 guide pratique pour le mesurage sur le lieu de travail

NF EN1032+A1 :2009 «Essai des machines mobiles dans le but de déterminer la valeur d'émission vibratoire »

NF EN 12096 : 1997 « Déclaration et vérification des valeurs d'émission vibratoire »

ISO 2361-1+A1 :2010 « Évaluation de l'exposition des individus à des vibrations globales du corps Partie 1 : spécifications générales. »

ISO 10326/A1 \& A2 :2011 «Vibrations mécaniques Méthode en laboratoire pour l'évaluation des vibrations du siège de véhicule - Partie 1 : exigences de base». 